\title{
RELATIVE SCHUR-CONVEXITY ON GLOBAL NPC SPACES
}

\author{
Constantin P. Niculescu And IOnEL RovenţA
}

Abstract. We introduce the concept of relative convexity on spaces with global nonpositive curvature and illustrate its usefulness by a number of inequalities involving the convex functions on such spaces.

Mathematics subject classification (2010): Primary 52A40; Secondary 26B25, $26 \mathrm{D} 15$. Keywords and phrases: Global NPC space, convex function, stochastic matrix.

\section{REFERENCES}

[1] W. BALlmanN, Lectures on spaces with nonpositive curvature, DMV Seminar Band 25, Birkhäuser, Basel, 2005.

[2] R. Bhatia, Positive definite matrices, Princeton University Press, 2007.

[3] J. BorCEA, Equilibrium points of logarithmic potentials induced by positive charge distributions. I. Generalized de Bruijn-Springer relations, Trans. Amer. Math. Soc. 359 (2007), 3209-3237.

[4] M. R. BRIDSON AND A. HAEFliger, Metric spaces of non-positive curvature, Grundlehren der Mathematischen Wissenschaften vol. 319, Springer-Verlag, 1999.

[5] J. Eells And B. Fuglede, Harmonic maps between Riemannian polyhedra, Cambridge University Press, 2001

[6] G. H. Hardy, J. E. Littlewood and G. Pólya, Inequalities, Cambridge Mathematical Library, 2nd Edition, 1952, Reprinted 1988.

[7] J. Jost, Equilibrium maps between metric spaces, Calc. Var. 2 (1994), 173-204.

[8] J. Jost, Nonpositive curvature: geometric and analytic aspects, Lectures in Mathematics ETH Zürich, Birkhäuser Verlag, Basel, 1997.

[9] Y. LiM, Convex geometric means, J. Math. Anal. Appl. 404 (2013), No. 1, 115-128.

[10] A. W. Marshal, I. Olkin And B. C. ARnold, Inequalities: Theory of Majorization and Its Applications, 2nd Edition, Springer-Verlag, 2011.

[11] C. P. Niculescu AND L.-E. Persson, Convex Functions and their Applications. A Contemporary Approach, CMS Books in Mathematics vol. 23, Springer-Verlag, New York, 2006.

[12] C. P. Niculescu And I. RovenţA, An Approach of Majorization in Spaces with a Curved Geometry, J. Math. Anal. Appl. 411 (2014), No. 1, 119-128.

[13] C. P. Niculescu And I. RovenţA, Relative convexity and its applications, Aequationes Mathematicae, 2015, DOI: 10.1007/s00010-014-0319-X.

[14] J. PeČarić, F. Proschan, Y. L. Tong, Convex functions, partial orderings, and statistical applications, Academic Press, 1992.

[15] K. T. StURm, Probability measures on metric spaces of nonpositive curvature, In vol.: Heat kernels and analysis on manifolds, graphs, and metric spaces (Pascal Auscher et al. editors), Lecture notes from a quarter program on heat kernels, random walks, and analysis on manifolds and graphs, April 16-July 13, 2002, Paris, France. Contemp. Math. 338 (2003), 357-390. 